\title{
DIMENSIONS OF TOPOLOGICAL GROUPS CONTAINING THE BOUQUET OF TWO CIRCLES
}

\author{
TAKASHI KIMURA
}

(Communicated by Dennis Burke)

\begin{abstract}
In this paper we prove the following: If a topological group $G$ contains the bouquet $S^{1} \vee S^{1}$, then $\operatorname{dim} G \geq 2$ holds. This is a counterexample to a question of $\mathrm{Bel}^{\prime}$ nov in the one-dimensional case.
\end{abstract}

\section{INTRODUCTION}

In this paper we assume that all spaces are Tychonoff.

Bel'nov [1] proved that every space $X$ can be embedded into a homogeneous space $G_{X}$ such that ind $G_{X}=\operatorname{ind} X, \operatorname{Ind} G_{X}=\operatorname{Ind} X$, and $\operatorname{dim} G_{X}=\operatorname{dim} X$ in the case when the corresponding dimension of $X$ is finite. The space $G_{X}$ is a free group over $X$ with continuous right translations. Bel'nov (see [5]) asked whether every space $X$ can be embedded into a topological group $G$ with $\operatorname{dim} G \leq \operatorname{dim} X$.

Shakhmatov [5] proved that if $n \neq 0,1,3,7$, then the $n$-dimensional sphere $S^{n}$ cannot be embedded into an $n$-dimensional topological group. Thus the answer to the above question is no. However, Shakhmatov also proved that in case $\operatorname{dim} X=0$ the answer to this question is yes. Hence it is natural to ask whether every space $X$ can be embedded into a topological group $G$ with $\operatorname{dim} G \leq \operatorname{dim} X$ in case $\operatorname{dim} X=1$ or 3 or 7 .

In this paper we prove that the answer to this question is no in case $\operatorname{dim} X=$ 1. Namely, we prove that if a topological group $G$ contains the bouquet $S^{1} \vee$ $S^{1}$, then $\operatorname{dim} G \geq 2$ holds. Obviously, $\operatorname{dim}\left(S^{1} \vee S^{1}\right)=1$. Hence the bouquet $S^{1} \vee S^{1}$ is a counterexample to a question of Bel'nov in the one-dimensional case.

\section{Preliminaries AND lemmas}

We denote by $\mathbb{R}, \mathbb{Q}, I$, and $S^{1}$ the real line, the rational space, the closed unit interval $[0,1]$, and the 1 -sphere, respectively. We put $\partial I^{2}=(I \times\{0,1\}) \cup$ $(\{0,1\} \times I)$. We denote by $d$ the Euclidean metric on $\mathbb{R}^{3}$. For two continuous

Received by the editors January 16, 1990 and, in revised form, November 5, 1990.

1980 Mathematics Subject Classification (1985 Revision). Primary 54F45, 54C25; Secondary $05 \mathrm{C} 10,22 \mathrm{~A} 05$.

Key words and phrases. Topological group, dimension, embedding, bouquet, graph. 
mappings $f$ and $g$ of a space $X$ into $\mathbb{R}^{3}$ we put

$$
d(f, g)=\sup \{d(f(x), g(x)): x \in X\} \text {. }
$$

For a subset $X$ of $\mathbb{R}^{3}$ and for any $\varepsilon>0$ we put

$$
U(X, \varepsilon)=\left\{\alpha \in \mathbb{R}^{3}: d(\alpha, X)<\varepsilon\right\} .
$$

For a subset $X$ of $\mathbb{R}^{3}$ we denote by $i_{X}$ the inclusion mapping of $X$ into $\mathbb{R}^{3}$. For a mapping $f: X \rightarrow Y$ and for a subset $A$ of $X$ we denote by $f_{\mid A}$ the restriction of $f$ to $A$. Let $N_{1}^{3}$ be the subspace of $\mathbb{R}^{3}$ consisting of all points which have at most one rational coordinate. The following lemma is needed in $\S 3$.

2.1. Lemma. Let $X$ be a compact metrizable space with $\operatorname{dim} X \leq 1$. Then for every continuous mapping $f: X \rightarrow \mathbb{R}^{3}$ and for any $\varepsilon>0$ there exists an embedding $g: X \rightarrow \mathbb{R}^{3}$ such that $d(f, g)<\varepsilon$ and $g(X) \subset N_{1}^{3}$.

Proof. See the proof of $[2,1.11 .5]$.

Let us set

$$
\begin{aligned}
L & =\left\{(x, y, 0) \in \mathbb{R}^{3}: x^{2}+y^{2}=9\right\}, \\
M & =\left\{(x, 0, z) \in \mathbb{R}^{3}:(x-2)^{2}+z^{2}=1\right\}, \\
C & =\left\{(x, y, 0) \in \mathbb{R}^{3}: x^{2}+y^{2}=4\right\}, \\
Z & =\left\{(0,0, z) \in \mathbb{R}^{3}: z \in \mathbb{R}\right\}, \\
\mathbb{T}^{2} & =\left\{\alpha \in \mathbb{R}^{3}: d(\alpha, C)=1\right\} .
\end{aligned}
$$

Then, obviously, $\mathbb{T}^{2}$ is a torus. For every $\theta, 0 \leq \theta<2 \pi$, we put

$$
F_{\theta}=\left\{(r \cos \theta, r \sin \theta, z) \in \mathbb{R}^{3}: r>0 \text { and } z \in \mathbb{R}\right\} \text {. }
$$

Let $K$ be a polyhedral 1-sphere satisfying the following two conditions $(*)$ :

$$
\begin{aligned}
& K \subset U(C, 1) \cap(((\mathbb{R} \times \mathbb{Q}) \cup(\mathbb{Q} \times \mathbb{R})) \times\{0\}) \quad \text { and } \\
& \left|F_{\theta} \cap K\right|=1 \quad \text { for every } \theta, 0 \leq \theta<2 \pi .
\end{aligned}
$$

We shall construct a continuous mapping $p_{K}: \mathbb{R}^{3}-(K \cup Z) \rightarrow \mathbb{T}^{2}$. For every $\theta$, $0 \leq \theta<2 \pi$, let $p_{\theta}: F_{\theta}-\left\{x_{\theta}\right\} \rightarrow F_{\theta} \cap \mathbb{T}^{2}$ be the projection of $F_{\theta}-\left\{x_{\theta}\right\}$ from the point $x_{\theta}$ onto $F_{\theta} \cap \mathbb{T}^{2}$, where $\left\{x_{\theta}\right\}=F_{\theta} \cap K$. Let $p_{K}: \mathbb{R}^{3}-(K \cup Z) \rightarrow \mathbb{T}^{2}$ be the mapping defined by

$$
p_{K}(r \cos \theta, r \sin \theta, z)=p_{\theta}(r \cos \theta, r \sin \theta, z)
$$

for every $(r \cos \theta, r \sin \theta, z) \in \mathbb{R}^{3}-(K \cup Z)$. Then $p_{K}$ is continuous. The proof of the following lemma is easy, so we omit the proof.

2.2. Lemma. For every $\varepsilon>0$ there exists a polyhedral 1-sphere $K$ with property $(*)$ such that $d\left(i_{U\left(\mathbb{T}^{2}, \varepsilon\right)}, p_{K \mid U\left(\mathbb{T}^{2}, \varepsilon\right)}\right)<2 \varepsilon$.

Let $\lambda: I^{2} \rightarrow \mathbb{T}^{2}$ be the natural quotient such that

$$
\begin{aligned}
& \lambda(x, 0)=\lambda(x, 1)=(3 \cos 2 \pi x, 3 \sin 2 \pi x, 0), \\
& \lambda(0, x)=\lambda(1, x)=(2+\cos 2 \pi x, 0, \sin 2 \pi x),
\end{aligned}
$$

and

$$
\lambda(1 / 2,1 / 2)=(-1,0,0)
$$


Let $q: I^{2}-\{(1 / 2,1 / 2)\} \rightarrow \partial I^{2}$ be the projection of $I^{2}-\{(1 / 2,1 / 2)\}$ from the point $(1 / 2,1 / 2)$ onto $\partial I^{2}$. It is obvious that $\left|\lambda q \lambda^{-1}(\alpha)\right|=1$ for every $\alpha \epsilon$ $\mathbb{T}^{2}-\{(-1,0,0)\}$. Thus we can define the mapping $\mu: \mathbb{T}^{2}-\{(-1,0,0)\} \rightarrow \mathbb{T}^{2}$ such that $\{\mu(\alpha)\}=\lambda q \lambda^{-1}(\alpha)$. Then we have $\mu\left(\mathbb{T}^{2}-\{(-1,0,0)\}\right)=L \cup M$ and $\mu$ is continuous. The proof of the following lemma is also easy, so we omit the proof.

2.3. Lemma. For every $\varepsilon>0$ there exists $\delta>0$ such that

$$
d\left(i_{\left.U(L \cup M, \delta) \cap \mathbb{T}^{2}\right)}, \mu_{\mid U(L \cup M, \delta) \cap \mathbb{T}^{2}}, \mu_{\left|U(L \cup M, \delta) \cap T^{2}\right|}\right)<\varepsilon .
$$

\section{THE MAIN THEOREM}

By Lemma 2.3, we can take $\delta>0$ such that

$$
d\left(i_{U(L \cup N, \delta) \cap \mathbb{T}^{2}}, \mu_{\mid U(L \cup M, \delta) \cap \mathbb{T}^{2}}\right)<1 / 3 .
$$

We fix $\delta$ in this section.

3.1. Lemma. Let $f: I^{2} \rightarrow \mathbb{R}^{3}$ be a continuous mapping such that $d\left(f_{\mid \partial I^{2}}, \lambda_{\mid \partial I^{2}}\right)$ $<\delta / 4$. Then $\operatorname{dim} f\left(I^{2}\right) \geq 2$ holds.

Proof. Suppose that $\operatorname{dim} f\left(I^{2}\right) \leq 1$. By Lemma 2.1, we can take an embedding $g: f\left(I^{2}\right) \rightarrow \mathbb{R}^{3}$ such that $d\left(i_{f\left(I^{2}\right)}, g\right)<\delta / 4$ and $g \circ f\left(I^{2}\right) \subset N_{1}^{3}$. By Lemma 2.2 , we can take a polyhedral 1 -sphere $K$ with property $(*)$ such that $d\left(i_{U\left(\mathbb{T}^{2}, \delta / 2\right)}, p_{K \mid U\left(\mathbb{T}^{2}, \delta / 2\right)}\right)<\delta$. Note that $g \circ f\left(I^{2}\right) \cap(K \cup Z)=\varnothing$, because $g \circ f\left(I^{2}\right) \subset N_{1}^{3}$. Since $K \subset((\mathbb{R} \times \mathbb{Q}) \cup(\mathbb{Q} \times \mathbb{R})) \times\{0\}$ and $g \circ f\left(I^{2}\right) \cap([-3,0] \times$ $\{(0,0)\})=\varnothing$, we have $(-1,0,0) \notin p_{K} \circ g \circ f\left(I^{2}\right)$. Let $\nu=\mu \circ p_{K} \circ g \circ f$. Then $\nu$ is a continuous mapping of $I^{2}$ into $\mathbb{T}^{2}$ such that $\nu\left(I^{2}\right) \subset L \cup M$ and $d\left(\nu_{\mid \partial I^{2}}, \lambda_{\partial I^{2}}\right)<1 / 3$. We take a lifting $\tilde{\nu}: I^{2} \rightarrow \mathbb{R}^{2}$ of $\nu$. Let us set $A_{i}=I \times\{i\}$ for $i=0,1$, and $B_{i}=\{i\} \times I$ for $i=0,1$. Since $d\left(\nu_{\mid \partial I^{2}}, \lambda_{\mid \partial I^{2}}\right)<1 / 3$, we may assume that

and

$$
\tilde{\nu}\left(A_{i}\right) \subset V\left(A_{i}, 1 / 3\right) \text { for } i=0,1 \text {, }
$$

$$
\tilde{\nu}\left(B_{i}\right) \subset V\left(B_{i}, 1 / 3\right) \text { for } i=0,1,
$$

where $V(N, \varepsilon)$ is the $\varepsilon$-neighborhood of $N$ in $\mathbb{R}^{2}$. Let us set $D=\mathbb{R} \times\{1 / 2\}$ and $E=\{1 / 2\} \times \mathbb{R}$. Then $D$ is a partition in $\mathbb{R}^{2}$ between $\tilde{\nu}\left(A_{0}\right)$ and $\tilde{\nu}\left(A_{1}\right)$, and $E$ is a partition in $\mathbb{R}^{2}$ between $\tilde{\nu}\left(B_{0}\right)$ and $\tilde{\nu}\left(B_{1}\right)$. Thus $\tilde{\nu}^{-1}(D)$ is a partition in $I^{2}$ between $A_{0}$ and $A_{1}$, and $\tilde{\nu}^{-1}(E)$ is a partition in $I^{2}$ between $B_{0}$ and $B_{1}$. Thus we have $\tilde{\nu}^{-1}(D) \cap \tilde{\nu}^{-1}(E) \neq \varnothing$. On the other hand, since $\nu\left(I^{2}\right) \subset L \cup M$, we have $\tilde{\nu}\left(I^{2}\right) \subset p^{-1}(L \cup M)$, where $p: \mathbb{R}^{2} \rightarrow \mathbb{T}^{2}$ is the covering mapping. Thus we have $D \cap E \cap \tilde{\nu}\left(I^{2}\right)=\varnothing$. This implies that $\tilde{\nu}^{-1}(D) \cap \tilde{\nu}^{-1}(E)=$ $\varnothing$. This is a contradiction. Hence we have $\operatorname{dim} f\left(I^{2}\right) \geq 2$. Lemma 3.1 has been proved.

Recall that the bouquet $S^{1} \vee S^{1}$ is the one point union of $S^{1}$ and $S^{1}$ with the common point.

We are now in a position to establish our main theorem.

3.2. Theorem. If a topological group $G$ contains the bouquet $S^{1} \vee S^{1}$, then $\operatorname{dim} G \geq 2$ holds.

Proof. Suppose that a topological group $G$ with $\operatorname{dim} G \leq 1$ contains the bouquet $S^{1} \vee S^{1}$. Since $L \cup M$ is homeomorphic to $S^{1} \vee S^{1}$ and since $G$ is 
homogeneous, we may assume that $e=(3,0,0) \in L \cup M \subset G$, where $e$ is the neutral element of $G$. Let $\varphi_{1}: I \rightarrow G$ and $\varphi_{2}: I \rightarrow G$ be the mappings defined by

$$
\varphi_{1}(x)=(3 \cos 2 \pi x, 3 \sin 2 \pi x, 0)
$$

and

$$
\varphi_{2}(x)=(2+\cos 2 \pi x, 0, \sin 2 \pi x)
$$

for every $x \in I$. Let $\varphi: I^{2} \rightarrow G$ be the mapping defined by $\varphi(x, y)=\varphi_{1}(x)$. $\varphi_{2}(y)$ for every $(x, y) \in I^{2}$, where - is the group composition of $G$. Then $\varphi\left(I^{2}\right)$ is compact and metrizable. Since $\varphi_{1}(0)=\varphi_{1}(1)=\varphi_{2}(0)=\varphi_{2}(1)=$ $(3,0,0)=e$, we have $\varphi_{\mid \partial I^{2}}=\lambda_{\partial I^{2}}$. Let $f: \varphi\left(I^{2}\right) \rightarrow \mathbb{R}^{3}$ be a continuous mapping such that $f_{\mid \varphi\left(\partial I^{2}\right)}=i_{L \cup M}$. Since $\operatorname{dim} G \leq 1$, we have $\operatorname{dim} \varphi\left(I^{2}\right) \leq 1$. Thus, by Lemma 2.1 , we can take an embedding $g: \varphi\left(I^{2}\right) \rightarrow \mathbb{R}^{3}$ such that $d(f, g)<\delta / 4$. Then we have $d\left(g \circ \varphi_{\mid \partial I^{2}}, \lambda_{\mid \partial I^{2}}\right)<\delta / 4$. Thus, by Lemma 3.1, we have $\operatorname{dim} \varphi\left(I^{2}\right)=\operatorname{dim} g \circ \varphi\left(I^{2}\right) \geq 2$. This is a contradiction. Theorem 3.2 has been proved.

\section{REMARKS AND QUESTIONS}

4.1 Remark. Vopenka ([7] or see [3, 18-11]) constructed a compact space $X$ such that $\operatorname{dim} X=n<\omega$ and ind $X=\infty$. Shakhmatov [4] proved that the above space $X$ cannot be embedded into a finite-dimensional, locally pseudocompact topological group. However, it is unknown whether the above space $X$ can be embedded into a topological group with the same dimension of $X$.

4.2. Remark. In the proof of Theorem 3.2 we get a contradiction to assume that $\operatorname{dim} \varphi\left(I^{2}\right) \leq 1$. Because $\varphi\left(I^{2}\right)$ is compact and metrizable, if ind $G \leq 1$ or Ind $G \leq 1$, then we have $\operatorname{dim} \varphi\left(I^{2}\right) \leq 1$. Hence the bouquet $S^{1} \vee S^{1}$ cannot be embedded into a topological group $G$ such that ind $G \leq 1$ or Ind $G \leq 1$ or $\operatorname{dim} G \leq 1$.

It is well known that every separable metrizable space $X$ with $\operatorname{dim} X=n$ can be embedded into the $(2 n+1)$-dimensional cube $I^{2 n+1}$. Obviously, $I^{2 n+1}$ can be embedded into the $(2 n+1)$-dimensional torus $\mathbb{T}^{2 n+1}$. Hence every separable metrizable space $X$ with $\operatorname{dim} X=n$ can be embedded into a $(2 n+1)$ dimensional compact metrizable topological group. It is easy to see that every $n$ dimensional, locally separable metrizable space can be embedded into a $(2 n+1)$ dimensional metrizable topological group.

4.3. Question. Does there exist a mapping $\varphi: \omega \rightarrow \omega$ such that every metrizable space $X$ with $\operatorname{dim} X=n$ can be embedded into a (metrizable) topological group $G$ with $\operatorname{dim} G \leq \varphi(n)$ ?

Shakhmatov also gave some similar problems in [6].

It is obvious that the bouquet $S^{1} \vee S^{1}$ can be embedded into the 2-dimensional torus $\mathbb{T}^{2}$. However, it is well known that if $n \geq 8$, then the complete graph $K_{n}$ cannot be embedded into $\mathbb{T}^{2}$. Since $K_{8}$ contains $S^{1} \vee S^{1}, K_{n}$ cannot be embedded into a topological group $G$ with $\operatorname{dim} G \leq 1$ for each $n \geq 8$. Obviously, $K_{n}$ can be embedded into the 3-dimensional torus $\mathbb{T}^{3}$. 
4.4. Question. Let $n \geq 8$. Can the complete graph $K_{n}$ be embedded into a topological group $G$ with $\operatorname{dim} G \leq 2$ ?

A space $X$ is atriodic if $X$ does not contain the complete bipartite graph $K_{1,3}$. Obviously, the bouquet $S^{1} \vee S^{1}$ is not atriodic.

4.5. Question. Can the complete bipartite graph $K_{1,3}$ be embedded into a topological group $G$ with $\operatorname{dim} G=1$ ?

If a graph $X$ is atriodic, then every component of $X$ is homeomorphic to $S^{1}$ or $I$ or the singleton. Thus an atriodic graph can be embedded into $S^{1} \times \mathbb{Z}_{p}$, where $p$ is the number of components of $X$ and $\mathbb{Z}_{p}$ is a group consisting of $p$ points with discrete topology. Hence if the answer to the above question is negative, then a graph $X$ can be embedded into a topological group $G$ with $\operatorname{dim} G=1$ if and only if $X$ is atriodic.

Added in proof. $\mathrm{H}$. Kato and J. Kulesza, independently, constructed an $n$ dimensional space $X$ such that every topological group $G$ containing $X$ has $\operatorname{dim} G \geq n+1$. They also gave a negative answer to Question 4.5.

\section{REFERENCES}

1. V. K. Bel'nov, Dimension of topologically homogeneous spaces and free homogeneous spaces, Soviet Math. Dokl. 19 (1978), 86-89.

2. R. Engelking, Dimension theory, Polish Scientific, Warszawa, 1977.

3. K. Nagami, Dimension theory, Academic Press, New York and London, 1970.

4. D. B. Shakhmatov, Closed embeddings into pseudocompact spaces preserving the covering dimension, Vestnik Moskov. Univ. Ser. I Mat. Mekh. 43 (1988), 69-71.

5. __ Imbedding into topological groups preserving dimensions, Topology Appl. 36 (1990), 181-204.

6. _ A survey of current researches and open problems in the dimension theory of topological groups, Questions Answers Gen. Topology 8 (1990), 101-118.

7. P. Vopenka, On the dimension of compact spaces, Czechoslovak Math. J. 8 (1958), 319-327.

Institute of Mathematics, University of Tsukuba, Tsukuba-Shi, Ibaraki, 305, JaPan

Current address: Department of Mathematics, Faculty of Education, Saitama University, Urawa, Saitama 338 Japan 